\title{
Effect of Random Errors in Planar Near-Field Measurement
}

\author{
ALLEN C. NEWELL, SENIOR MEMBER, IEEE, AND CARL F. STUBENRAUCH, SENIOR MEMBER, IEEE
}

\begin{abstract}
Expressions which relate the signal-to-noise ratio in the near field to the signal-to-noise ratio $(S / N)$ in the far field are developed. The expressions are then used to predict errors in far-field patterns obtained from near-field data. A technique to measure the noise in the far-field pattern is also given.
\end{abstract}

\section{INTRODUCTION}

$\mathrm{N}$ EAR-FIELD measurements have been employed for a number of years to obtain far-field patterns for antennas from data acquired on a surface (plane, cylinder, or sphere). Of these techniques, the planar technique is perhaps the most fully developed. For this technique, a detailed analysis of errors due to receiver and the probe positioning mechanism has been performed [1], [2]. For most applications, these systematic errors are dominant in determining the overall accuracy of the resulting far-field measurement. Recent extensions of the near-field method to the measurement of low-sidelobe and other high-performance antennas and the required improvement of near-field measurement facilities have caused rethinking of the question of random errors. The purpose of this paper is to analyze the effect of random errors in the measurement of the amplitude and phase of the near field. These errors may arise from the digitization of the data and receiver noise.

We first review the basic equations which describe the relation between the near fields and the far fields of the antenna under test (AUT). A model for random errors will then be developed from which estimates of the errors in the near field of the antenna are obtained. This model will include both Gaussian receiver noise and the effect of digitization which is uniformly distributed. We then apply the model to a case of an actual antenna and simulate the errors obtaining as a result the signal-to-noise ratio $(\mathrm{S} / \mathrm{N})$ in the calculated far field defined as the ratio of the maximum value of the far field to the noise power in the far field. Finally, we describe a method of measuring the noise which appears in the far field based on actual measurements and compare it to noise in the near field.

\section{Basic Relationships for Near-Field Antenna MEASUREMENTS}

The theory of planar near-field measurements has been well developed elsewhere [3]. In this section, we will give only those relationships which will be used in the development of the error expressions.

Manuscript received September 21, 1987; revised January 21, 1988.

The authors are with the Electromagnetic Fields Division, National Bureau of Standards, Boulder, $\mathrm{CO} 80303$.

IEEE Log Number 8821100
In planar near-field scanning, the insertion loss between the probe antenna and the AUT is measured on a raster pattern at a spacing determined by the sampling theorem. The relation between the measured near field and the antenna pattern is given by the Fourier transform relationship,

$$
b_{0}^{\prime}(\mathbf{P}) / a_{0}=F^{\prime} \int \mathbf{t}_{10}(\mathbf{K}) \cdot s_{02}^{\prime}(\mathbf{K}) e^{i \gamma d} e^{i \mathbf{K} \cdot \mathbf{P}} d k_{x} d k_{y}
$$

where $b_{0}^{\prime}$ is the complex wave amplitude emergent from the probe antenna, $a_{0}$ is the complex wave amplitude incident on the AUT, $\mathbf{P}=x \mathbf{e}_{x}+y \mathbf{e}_{y}$ is the transverse position of the probe antenna in the scan plane, and $d$ is the separation between the probe antenna and the scan plane. $\mathbf{K}=k_{x} \mathbf{e}_{x}+$ $k_{y} \mathbf{e}_{y}$ is the transverse component of the propagation vector, $\gamma$ is the longitudinal component, $\mathbf{t}_{10}$ is the transmitting characteristic of the AUT, $\mathbf{s}_{02}^{\prime}$ is the receiving characteristic of the probe, and $F^{\prime}$ is a mismatch factor. The transmitting and receiving characteristics of the AUT and probe are simply related to their far fields.

The quantity $\mathbf{t}_{10} \cdot \mathbf{s}_{02}^{\prime}$ is often designated $D(\mathbf{K})$ and is in fact a single component of the far field of the AUT if the probe is ideal, i.e., a probe which measures a single component of the electric field at a point. It is this quantity for which we will determine the errors. Inverting (1) gives the expression for $D(\mathbf{K})$ in terms of the measured insertion loss,

$$
D(\mathbf{K})=\frac{e^{-i \gamma d}}{4 \pi^{2} F^{\prime} A} \int B(\mathbf{P}) e^{-i \mathbf{K} \cdot \mathbf{P}} d x d y
$$

where we have let $B(\mathbf{P})$ be the normalized probe output $b_{0}^{\prime}(\mathbf{P}) / b_{0}^{\prime}\left(\mathbf{P}_{0}\right)$ and $A$ is a normalizing constant given by $a_{0} /$ $b_{0}^{\prime}\left(\mathbf{P}_{0}\right)$. By virtue of the exponential, $D(\mathbf{K})$ is for all practical purposes spatially band-limited, hence the sampling theorem may be applied to evaluate the integral in (2) without error using the following sum,

$$
D(\mathbf{K})=\frac{e^{-i \gamma d}}{4 \pi^{2} F^{\prime} A} \delta_{x} \delta_{y} \sum B\left(\mathbf{P}_{r s}\right) e^{-i \mathbf{K} \cdot \mathbf{P}_{r s}}
$$

where $\delta_{x}$ and $\delta_{y}$ are the near-field data point spacings in the $x$ and $y$-directions. This summation may be conveniently evaluated using the FFT algorithm.

\section{MODEL FOR ERRORS}

We briefly outline the analysis below. The error-free output of the probe may be expressed in terms of amplitude and phase by

$$
B(\mathbf{P}) \equiv a(\mathbf{P}) e^{i \psi(\mathbf{P})} .
$$


The measurement of the amplitude $a(\mathbf{P})$ and phase $\psi(\mathbf{P})$ will introduce some errors which may be denoted by $\Delta a(\mathbf{P})$ and $\Delta \psi(\mathbf{P})$. Thus the amplitude $a_{m}$ and phase $\psi_{m}$ indicated by the receiver will be

$$
a_{m}(\mathbf{P})=a(\mathbf{P})+\Delta a(\mathbf{P}) \quad \psi_{m}(\mathbf{P})=\psi(\mathbf{P})+\Delta \psi(\mathbf{P})
$$

The quantities $\Delta a(\mathbf{P})$ and $\Delta \psi(\mathbf{P})$ represent the errors as they are most commonly given by the receiver manufacturers. The error in the measured data is $\epsilon(\mathbf{P})$ which is given by

$$
\begin{aligned}
\epsilon(\mathbf{P}) & =[a(\mathbf{P})+\Delta a(\mathbf{P})] e^{i(\psi(\mathbf{P})+\Delta \psi(\mathbf{P}))}-a(\mathbf{P}) e^{i \psi(\mathbf{P})} \\
& =\left[a(\mathbf{P})\left(e^{i \Delta \psi(\mathbf{P})}-1\right)+\Delta a(\mathbf{P}) e^{i \Delta \psi(\mathbf{P})}\right] e^{i \psi(\mathbf{P})} .
\end{aligned}
$$

We now expand the exponential phase factor and retain only terms which are second order or less, hence

$$
\begin{aligned}
\epsilon(\mathbf{P}) \approx[\Delta a(\mathbf{P})+ & i a(\mathbf{P}) \Delta \psi(\mathbf{P}) \\
& \left.+i \Delta a(\mathbf{P}) \Delta \psi(\mathbf{P})-a(\mathbf{P}) \Delta \psi^{2}(\mathbf{P}) / 2\right] e^{i \psi(\mathbf{P})} .
\end{aligned}
$$

The quantity of interest is the spectrum $D(\mathbf{K})$ which is proportional to the far-field pattern in planar scanning given by (2). Because of the linearity of the Fourier transform, the spectrum of the error will be given by

$$
\begin{aligned}
\Delta D(\mathbf{K})= & \frac{e^{-i \gamma d}}{4 \pi^{2} F^{\prime} A} \int \epsilon(\mathbf{P}) e^{-i \mathbf{K} \cdot \mathbf{P}} d x d y \\
= & C \int[\Delta a(\mathbf{P})+i a(\mathbf{P}) \Delta \psi(\mathbf{P})+i \Delta a(\mathbf{P}) \Delta \psi(\mathbf{P}) \\
& \left.-a(\mathbf{P}) \Delta \psi^{2}(\mathbf{P}) / 2\right] e^{i[\psi(\mathbf{P})-\mathbf{K} \cdot \mathbf{P}]} d x d y .
\end{aligned}
$$

We begin by considering the effect of the amplitude error term in (7). We combine the factors $\psi(\mathbf{P})$ and $\mathbf{K} \cdot \mathbf{P}$ into a net phase factor $\Phi(\mathbf{K}, \mathbf{P})$ and express the integral as a summation. Thus the amplitude error term (the first one) has the form

$\Delta D_{a}(\mathbf{K})=C \delta_{x} \delta_{y} \sum[\Delta a(\mathbf{P}) \cos \Phi(\mathbf{K}, \mathbf{P})$

$$
+i \Delta a(\mathbf{P}) \sin \Phi(\mathbf{K}, \mathbf{P})]
$$

Now in the direction near the main beam, the factor $\Phi(\mathbf{K}, \mathbf{P})$ is approximately constant. However, in the sidelobe region, the random amplitude error will be modulated by the sine and cosine factors. Therefore, the summation may be estimated by

$$
\sum_{i=1}^{N} \Delta a_{i}(\mathbf{P}) \cos \Phi(\mathbf{K}, \mathbf{P}) \leq \sum_{i=1}^{N} \Delta a_{i}(\mathbf{P}) \leq N\left(M_{a} \pm 3 \sigma_{m a}\right)
$$

where

$M_{a}$ mean of the distribution of $\Delta a(\mathbf{P}) \cos \Phi(\mathbf{K}, \mathbf{P})$,

$\sigma_{m a}$ standard deviation of the mean,

$N$ total number of data points.

The summation involving the sine term is similarly estimated.

In the off-axis region, $M_{a}=0$. Further, the standard deviation of the mean is related to the standard deviation $\sigma_{a}$ of the amplitude error distribution by

$$
\sigma_{m a}=\frac{\sigma_{a}}{\sqrt{N}}
$$

hence

$$
\left|\Delta D_{a}(\mathbf{K})\right| \leq \frac{3 \sqrt{2} \sqrt{N}}{4 \pi^{2} F^{\prime} A} \delta_{x} \delta_{y} \sigma_{a} .
$$

The on-axis value of the $D(\mathbf{K})$ may be approximated with good accuracy by

$$
\left|D\left(\mathbf{K}_{0}\right)\right|=\frac{A_{e}}{4 \pi^{2} F^{\prime} A}=\frac{A_{a} \eta}{4 \pi^{2} F^{\prime} A}
$$

where $A_{e}$ is the effective area of the antenna, $A_{a}$ is the physical area of the antenna, and $\eta$ is the aperture efficiency. The $\mathrm{S} / \mathrm{N}$ in the far field may thus be expressed as

$$
\left|\frac{D\left(\mathbf{K}_{0}\right)}{\Delta D_{a}}\right|=\frac{N_{e}}{3 \sqrt{2} \sigma_{a} \sqrt{N}}=\frac{\sqrt{N_{e}}}{3 \sqrt{2} \sigma_{a} \sqrt{N / N_{e}}} .
$$

Here, we have chosen to express the relationship in terms of $N_{e}$, the number of data points within the effective aperture of the antenna. The second form of the equation has been included for later comparison with the expression for the phase errors.

The analysis for phase errors requires that two separate cases be considered depending on the region of interest in the far field. We first consider the case of the sidelobe region. Here the dominant term is the second one in (6), since the remaining two terms involving phase errors contain the product of two small terms. The error spectrum in the sidelobe region due to random phase errors is then

$$
\begin{aligned}
\Delta D_{\psi}(\mathbf{K})= & \frac{e^{-i \gamma d}}{4 \pi^{2} F^{\prime} A} \int i a_{t}(\mathbf{P}) \Delta \psi(\mathbf{P}) e^{i \Phi(\mathbf{K}, \mathbf{P})} d x d y \\
= & i C \delta_{x} \delta_{y} \sum\left[a_{t}\left(\mathbf{P}_{i}\right) \Delta \psi\left(\mathbf{P}_{i}\right) \cos \Phi\left(\mathbf{K}, \mathbf{P}_{i}\right)\right. \\
& \left.+i a_{t}\left(\mathbf{P}_{i}\right) \Delta \psi\left(\mathbf{P}_{i}\right) \sin \Phi\left(\mathbf{K}, \mathbf{P}_{i}\right)\right] .
\end{aligned}
$$

The amplitude factor $a_{t}$ is essentially zero outside the scan area. Further, because of the amplitude taper factor, the summation may be reduced to include only those points within the effective area of the antenna. The sum may thus be estimated in a similar fashion to (9)

$$
\begin{aligned}
\sum_{i=1}^{N} a_{t}(\mathbf{P}) \Delta \psi_{i}(\mathbf{P}) \cos \Phi(\mathbf{K}, \mathbf{P}) \\
\leq \sum_{i=1}^{N} a_{t}(\mathbf{P}) \Delta \psi_{i}(\mathbf{P}) \leq N_{e}\left(M_{\psi} \pm 3 \sigma_{m \psi}\right)
\end{aligned}
$$

where $\boldsymbol{M}_{\psi}$ is the mean of the distribution of $a_{t}(\mathbf{P}) \Delta \psi(\mathbf{P}) \cos$ $\Phi(\mathbf{K}, \mathbf{P})$ and $\sigma_{m \psi}$ is the standard deviation of the mean of $a_{t}(\mathbf{P})$ $\Delta \psi(\mathbf{P})$.

Thus the phase error spectrum can be represented most simply by

$$
\left|\Delta D_{\psi}(\mathbf{K})\right| \leq \frac{3 \sqrt{2}}{4 \pi^{2} F^{\prime} A} \sqrt{N_{e}} \delta_{x} \delta_{y} \sigma_{\psi} .
$$


Finally, we get the $\mathrm{S} / \mathrm{N}$ in the presence of phase errors only

$$
\left|\frac{D\left(\mathbf{K}_{0}\right)}{\Delta D_{\psi}}\right|=\frac{\sqrt{N_{e}}}{3 \sqrt{2} \sigma_{\psi}} .
$$

When both amplitude and phase errors are significant, the total error $\Delta D$ may be estimated as the root-mean-square of the individual errors $\Delta D_{a}$ and $\Delta D_{\psi}$ if the amplitude and phase errors are assumed independent. Thus the $\mathrm{S} / \mathrm{N}$ in the off-axis region is given by the relation

$$
\left|\frac{D\left(\mathbf{K}_{0}\right)}{\Delta D}\right|=\frac{\sqrt{N_{e}}}{3 \sqrt{2}} \frac{1}{\sqrt{\frac{N}{N_{e}} \sigma_{a}^{2}+\sigma_{\psi}^{2}}} .
$$

We now consider the effect of phase errors on the pattern within the region of the main beam. In this region, the phase factor $\Phi$ is approximately constant. Now a constant phase may be added to the overall phase $\Phi$ such that the phase error term $a_{t} \Delta \psi$ has an average value of zero. Under this condition, the second term in (6) no longer contributes. The remaining terms are of second order. Of these two, the fourth term will dominate since it is always positive, while the third term will have an average close to zero for the same reason as the second. Yaghjian's analysis [1] for systematic instrumentation phase errors is equally valid for random errors. Since our major interest is in the sidelobe region we only quote his result here [1, eq. 82$]$ :

$$
\left|\frac{D\left(\mathbf{K}_{0}\right)}{\Delta D_{\psi}}\right| \leq 2 /\left(\Delta \psi_{\max }\right)^{2} \approx 2 / 9 \sigma_{\psi}^{2}
$$

where we have estimated $\Delta \psi_{\max }$ by $3 \sigma_{\psi}$.

\section{Experimental Verification}

To verify the theory outlined above, several tests were performed on actual near-field data acquired at the National Bureau of Standards (NBS). Parameters of the antenna and near-field data are as follows:

$\begin{array}{ll}\text { reflector antenna diameter } & 1.2 \mathrm{~m} \\ \text { aperture efficiency } & 0.5 \\ \text { frequency } & 8.4 \mathrm{GHz} \\ x \text { and } y \text { near-field point spacing } & 2 \mathrm{~cm} \\ 128 \text { data points in } x \text { and } y . & \end{array}$

Patterns were computed with unperturbed amplitude and phase data and then recomputed with data which had been contaminated by a variety of techniques. For this study, the unperturbed data were considered to be without error. In order to simulate the effect of digitization, the data were rounded to the one-percent level in amplitude and/or to the nearest $1^{\circ}$ in phase. This corresponds to a random variable which is uniformly distributed between $\pm 1 / 2$ percent or $\pm 1 / 2^{\circ}$. (The standard deviation of a uniformly distributed random variable is given by $\sigma=w / \sqrt{3}$ where $w$ is the half-width of the distribution.) Next a uniformly distributed random variable having the same standard deviation as the rounded data was added to the amplitude and phase data. Finally, to simulate the effect of receiver noise, a normally distributed random variable with the same standard deviation as the rounded data was added to the amplitude and phase. In each case the difference between the far-field patterns computed with unperturbed and the corrupted near-field data was calculated and plotted. The peak value of this difference pattern then represents the $\mathrm{S} / \mathrm{N}$ in the computed results. This value was then compared to the $\mathrm{S} / \mathrm{N}$ predicted by the theory.

The cases studied are summarized below:

case 1 near-field data rounded to the nearest one percent in amplitude;

case 2 near-field data rounded to the nearest $1^{\circ}$ in phase; case 3 combination of cases 1 and 2;

case 4 amplitude and phase error uniformly distributed between $\pm 1 / 2$ percent and $\pm 1 / 2^{\circ}$;

case 5 amplitude and phase error normally distributed with the same standard deviation as cases 3 and 4 .

Patterns showing the difference between the corrupted and uncorrupted near-field data for case 3 are shown in Fig. 1. Fig. 2 shows the difference between the far-field patterns calculated from the data of Fig. 1. (Other cases are very similar.) The results are summarized in Table I. The results for cases 3-5 will probably yield similar results since the standard deviations are identical. The results shown indicate that the techniques developed for estimating the worst-case far-field $\mathrm{S} / \mathrm{N}$ are useful for estimating the effects of roundoff and random noise.

It is possible to make a direct measurement of the $\mathrm{S} / \mathrm{N}$ in the far-field pattern computed from near-field data using the bandlimited nature of the spectrum $D(\mathbf{K})$. The technique may most easily be applied using one-dimensional scans. An oversampled set of data is acquired using a spacing much smaller than $\lambda / 2$. A one-dimensional Fourier transform is then performed. The values of the spectrum calculated for $k_{x} / k$ greater than one represent results for the evanescent region. The actual antenna spectrum in the evanescent region has been attenuated to such a low value that it could not possibly be measured; for example, for $k_{x} / k=1.1$ and $d=6 \lambda$, the attenuation from the exponential factor is $150 \mathrm{~dB}$. The calculated spectrum for $k_{x} /$ $k>1$ is therefore due to errors in the measurements, and for large $k_{x}$ values, random errors will predominate. From this direct measurement of the $\mathrm{S} / \mathrm{N}$ in the one-dimensional spectrum, the corresponding $\mathrm{S} / \mathrm{N}$ in the two-dimensional data may be inferred. For instance, let the one-dimensional measurement be taken in the $x$ direction over a length $l_{x}^{\prime}$ with data point spacing $\delta_{x}^{\prime}$. The corresponding two-dimensional data will involve data point spacings $\delta_{x}$ and $\delta_{y}$ and scan lengths $l_{x}$ and $l_{y}$. The $\mathrm{S} / \mathrm{N}$ for the two-dimensional measurement will then be

$$
\left|\frac{D\left(\mathbf{K}_{0}\right)}{\Delta D_{a}}\right|_{2 D}=\left|\frac{D\left(\mathbf{K}_{0}\right)}{\Delta D_{a}}\right|_{1 D} \sqrt{\frac{l_{x} l_{y} \delta_{x}^{\prime}}{l_{x}^{\prime} \delta_{x} \delta_{y}}} .
$$

Fig. 3 illustrates data taken on an ultra-low sidelobe array antenna at a $z$ distance of $6 \lambda$ with a point spacing of $0.07 \lambda$. The observed $\mathrm{S} / \mathrm{N}$ is approximately $71 \mathrm{~dB}$ and corresponds to the level of $76 \mathrm{~dB}$ calculated using the theory discussed above. 


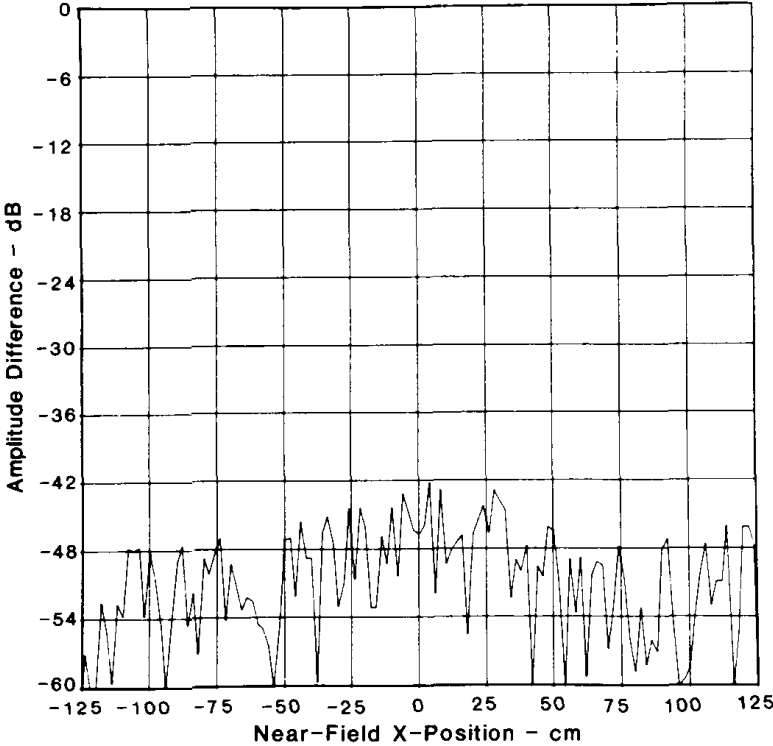

Fig. 1. Plot of difference between uncorrupted near-field data and nearfield data which was rounded to nearest one percent in amplitude and $1^{\circ}$ in phase. Note approximately $5-\mathrm{dB}$ increase in amplitude of pattern in vicinity of aperture of antenna. This corresponds to weighting of phase error by aperture taper factor.

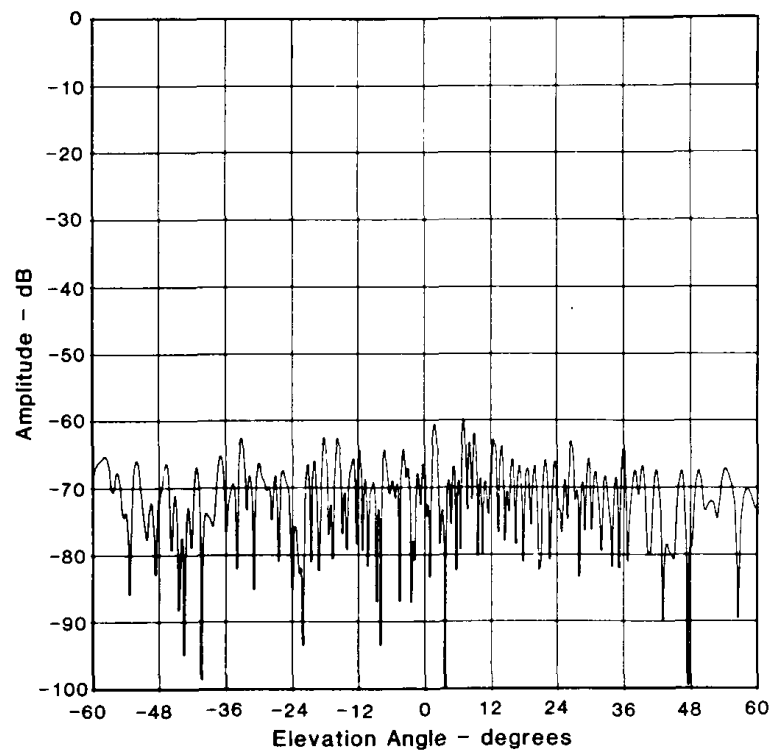

Fig. 2. Plot of difference between far-field pattern computed from uncorrupted near-field data and near-field data which was rounded to nearest one percent in amplitude and $1^{\circ}$ in phase. Antenna diameter: $1.2 \mathrm{~m}$; frequency: $4.0 \mathrm{GHz}$.

\section{CONCLUSION}

Random errors in antenna patterns computed from nearfield data may be significant if accurate results are needed in regions where pattern amplitudes are very low. In this paper we have shown how the effect of random errors may be estimated from the scan parameters and the noise level of the receiving equipment.
TABLE I

FAR FIELD S/N DUE TO RANDOM ERRORS IN NEAR-FIELD DATA

\begin{tabular}{ccccc}
\hline Case & $\sigma_{a}$ & $\sigma_{\psi}(\mathrm{rad})$ & $\begin{array}{c}\mathrm{S} / \mathrm{N}(\mathrm{dB}) \\
\text { (Calculated) }\end{array}$ & $\begin{array}{c}\mathrm{S} / \mathrm{N}(\mathrm{dB}) \\
(\text { Observed })\end{array}$ \\
\cline { 1 - 1 } 1 & 0.0029 & 0 & 59.2 & 61.0 \\
2 & 0 & 0.0050 & 65.9 & 71.0 \\
3 & 0.0029 & 0.0050 & 58.1 & 60.0 \\
4 & 0.0029 & 0.0050 & 58.1 & 62.5 \\
5 & 0.0029 & 0.0050 & 58.1 & 62.0
\end{tabular}

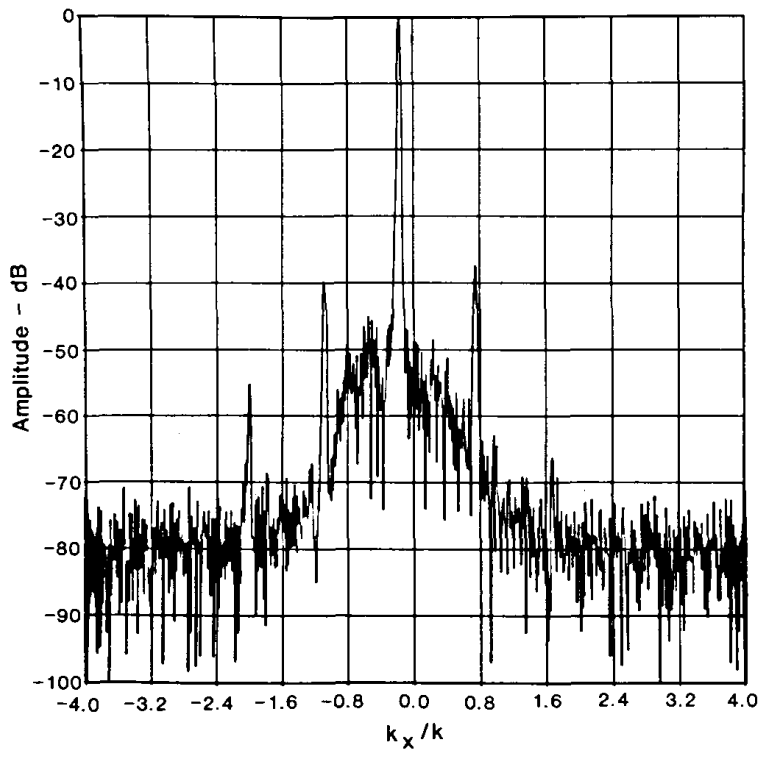

Fig. 3. Spectrum calculated into evanescent region $\left(k_{x} / k>1\right)$ using oversampled set of near-field data taken on low-sidelobe antenna. Values calculated in evanescent region correspond to noise rather than actual spectral values. Prominent sidelobes which occur at $k_{x} / k=-1.1,-2.0$, $0.75,1.65$ are artifacts of measurement system, result of multiple reflections.

We have shown that the effect on $\mathrm{S} / \mathrm{N}$ from random amplitude errors is proportional to the number of points in the effective area of the antenna, but inversely proportional to the square root of the total number of data points. Qualitatively, this may be understood by observing that random errors in the low-amplitude regions of the near field will add to degrade the overall $\mathrm{S} / \mathrm{N}$ while increasing the number of points taken in the effective area region will tend to average out errors.

Phase errors, on the other hand, have an effect only in the region where the near-field amplitude is high and are proportional to the square root of the number of data points taken in that area. In the main beam region, phase errors are proportional to the square of the phase error while the effect on the sidelobes is proportional to the first power of the phase error.

Various random errors were simulated by corrupting nearfield data and the resulting far-field patterns computed. Comparison between the simulation and the errors estimated by the appropriate formulas showed that the predicted error levels were within a few $\mathrm{dB}$ of the simulation. On the basis of this work we conclude that the formulas presented here do 
indeed provide a useful way of determining the effect of random near-field errors.

A technique for measuring the noise in the calculated farfield pattern by calculating the spectrum in the evanescent region from a single-dimensional oversampled scan has also been demonstrated with good results.

\section{REFERENCES}

[1] A. D. Yaghjian, "Upper-bound errors in far-field antenna parameters determined from planar near-field measurements, Part 1: Analysis," Nat. Bureau of Standards, Tech. Note 667, Oct. 1975

[2] A. C. Newell, "Upper-bound errors in far-field antenna parameters determined from planar near-field measurements, Part 2: Analysis and computer simulation," lecture notes for National Bureau of Standards short course. "Antenna Parameter Measurements by Near-Field Techniques," July 1975.

[3] D. M. Kerns, Plane-Wave Scattering-Matrix Theory of Antennas and Antenna-Antenna Interactions. Nat. Bureau of Standards, Monograph 162, June 1981.

Allen C. Newell (M 78-M 81-SM 83), for a photograph and biography please see page 733 of this issue.

Carl F. Stubenrauch (S'61-S'69-M'71-SM 83 ), for a photograph and biography please see page 733 of this issue. 